Check for updates

1 Department of Primary Care and Public Health, Imperial College London, London, UK

2 Population Health Research Institute, St George's University of London, London, UK

tasnime.osama15@imperial.ac.uk Cite this as: $B M J 2021 ; 373: n 861$ http://dx.doi.org/10.1136/bmj.n861

Published: 01 April 2021

\title{
Covid-19 vaccine passports: access, equity, and ethics
}

\author{
Governments should proceed with great caution through this minefield \\ Tasnime Osama, ${ }^{1}$ Mohammad S Razai, ${ }^{2}$ Azeem Majeed'
}

With millions of people receiving covid-19 vaccines globally, some countries have already started planning the implementation of "vaccine passports"-accessible certificates confirming covid-19 vaccination linked to the identity of the holder. The purpose of vaccine passports, governments argue, is to allow people to travel, attend large gatherings, access public venues, and return to work without compromising personal safety and public health. ${ }^{1}$ There remain, however, considerable practical and ethical challenges to their implementation.

Vaccine passports are not only permissible under international health regulations, they already exist. The World Health Organization endorses certificates confirming vaccination against yellow fever for entry into certain countries. ${ }^{2}$ Contrary to immunity passports, which may, perversely, incentivise infection, vaccine passports incentivise vaccination, ${ }^{3}$ an international public good with many positive benefits ${ }^{4}$ including individual and population immunity.

The public health principle of least infringement states that to achieve a public health goal, policy makers should implement the option that least impairs individual liberties. ${ }^{5}$ While lockdowns may be required, the continued restriction of the civil liberties of those who are immune and pose minimal risk of spreading infection may be unethical, as lack of freedom of movement is one of the most common adverse impacts of the pandemic on people's lives. ${ }^{36}$ Additionally, vaccine passports could help prevent other health and socioeconomic harms caused by lockdowns, thereby accruing individual and collective health, economic, and social benefits.

For vaccine passport holders to demonstrate protection from illness and lack of infectiousness, however, more evidence about the long term effectiveness of different types of vaccines and the duration of protection they confer is required, particularly with the regular emergence of new variants. The AstraZeneca vaccine may reduce transmission by up to $67 \%$ while the Pfizer BioNTech vaccine is $85 \%$ effective in preventing asymptomatic and symptomatic infections after the second dose, ${ }^{78}$ generating indirect benefits that extend to unvaccinated individuals through a reduction of SARS-CoV-2 circulation. Given that there are currently more than 200 vaccine trials underway, however, establishing the characteristics of each vaccine for the purpose of passport renewal would be challenging.

Vaccine passports need to be internationally standardised and must have verifiable credentials that safeguard against problems such as forgery and loss of privacy. WHO does not currently endorse covid-19 vaccine or immunity passports because of these concerns. ${ }^{9}$ It has, however, initiated a Smart Vaccination Certificate Working Group to establish key specifications and standards for effective and interoperable digital solutions for covid-19 vaccination.

Ethical concerns remain about the societal divide that these passports could cause. The Nuffield Council on Bioethics states that such passports could enable coercive and stigmatising workplaces, thereby compounding current structural disadvantages. ${ }^{10}$ Vaccine passports must be available and accessible to all to prevent exacerbating existing societal inequalities and worsening the health divide. Vaccines are scarce and access remains unequal, both globally and within countries. Covid-19 vaccines are also contraindicated in some people with serious health conditions and allergies. ${ }^{11}$ People facing vaccination access problems will be unable to obtain vaccine passports. Pregnant women are at an increased risk of severe covid-19 illness ${ }^{12}$; however, as clinical trials did not include pregnant women, the uncertain risk of vaccination during pregnancy may also lead to understandable hesitancy in this group. Ethnic minorities are also more likely to be vaccine hesitant. ${ }^{13}$

With most vaccine doses delivered in high income countries, WHO warned that the world is on the brink of a catastrophic moral failure. ${ }^{14}$ Because of vaccine nationalism and insufficient efforts to support globally coordinated access to covid-19 vaccines, nearly $25 \%$ of the world's population may not have access to a vaccine until at least 2022. ${ }^{15}$ This will widen the global north-south divide and create a situation where people from high income countries are able to travel, but not those from low income countries.

As vaccine passports would probably be digital and require access to private medical records, there are important questions around internet access, costs of acquiring and maintaining the passports, privacy, and data protection that must be tackled. Many consider adequate internet access a fundamental human right ${ }^{16}$; as large numbers of people do not have smartphones or stable internet connections, their exclusion breaches their rights to equality, particularly for those in low and middle income countries. Whether it is legal for workplaces, airlines, and entertainment and leisure venues to access vaccination data remains controversial, as this can perpetuate a form of elitism. ${ }^{17}$ Furthermore, ensuring that patient sensitive data are not used for other purposes is essential. 
While the merits of vaccine passports may be undeniable, implementation will require ethical justifications and practical solutions that do not discriminate against the poor, the less technically literate, and people from low and middle income countries. Without mitigation strategies and alternative solutions, the hardships experienced by marginalised and vulnerable groups will be intensified through the perpetuation of discrimination. If they are to be rolled out, the benefits of vaccine passports should not be dispersed unequally, and societies globally must strive to ensure that they are available to all.

Not commissioned, peer reviewed.

Competing interests: We have read and understood BMJ policy on declaration of interests and declare the following interests: none.

1 Royal Society. Twelve criteria for the development and use of covid-19 vaccine passports. 2021. https://royalsociety.org/-/media/policy/projects/set-c/set-c-vaccine-passports.pdf.

2 World Health Organization. Amendment to International Health Regulations (2005), annex 7 (yellow fever). 2013. www.who.int/ith/annex7-ihr.pdf.

3 Brown RCH, Kelly D, Wilkinson D, Savulescu J. The scientific and ethical feasibility of immunity passports. Lancet Infect Dis 2021;21:e58-63. doi: 10.1016/S1473-3099(20)30766-0 pmid: 33075284

4 Kaddar M. Economic characteristics of vaccines and immunizations. World Health Organization. www.who.int/influenza_vaccines_plan/resources/session_2_kaddar.pdf.

5 Chuan Voo T, Reis AA, Thome B, et al. Immunity certification for covid-19: ethical considerations. World Health Organization. 2021. www.who.int/bulletin/volumes/99/2/20-280701.pdf.

6 Office for National Statistics. Coronavirus and the social impacts on Great Britain: 11 December 2020.2020. www.ons.gov.uk/peoplepopulationandcommunity/healthandsocialcare/healthandwellbeing/bulletins/coronavirusandthesocialimpactsongreatbritain/11december2020\#impacts-onlife-and-well-being-of-adults-experiencing-some-form-of-depression-or-anxiety.

7 AstraZeneca. Covid-19 vaccine AstraZeneca confirms 100\% protection against severe disease, hospitalisation, and death in the primary analysis of phase III trials. 2021. www.astrazeneca.com/media-centre/press-releases/2021/covid-19-vaccine-astrazeneca-confirms-protection-against-severe-disease-hospitalisation-and-death-in-the-primary-analysis-of-phase-iiitrials.html.

8 Trial hints that Pfizer vaccine could curb covid transmission. Nature News 2021. www.nature.com/articles/d41586-020-00502-w.

9 Schlagenhauf P, Patel D, Rodriguez-Morales AJ, Gautret P, Grobusch MP, Leder K. Variants, vaccines and vaccination passports: Challenges and chances for travel medicine in 2021. Travel Med Infect Dis 2021;40:101996. doi: 10.1016/j.tmaid.2021.101996 pmid: 33631338

10 Nuffield Council on Bioethics. Rapid policy briefing: covid-19 antibody testing and "immunity certification." 2020. www.nuffieldbioethics.org/publications/covid-19-antibody-testing-and-immunity-certification.

11 Hassoun N. How to make "immunity passports" more ethical. Scientific American 2021. www.scientificamerican.com/article/how-to-make-immunity-passports-more-ethical.

12 Centers for Disease Control and Prevention. Vaccination considerations for people who are pregnant. 2021. www.cdc.gov/coronavirus/2019-ncov/vaccines/recommendations/pregnancy.html.

13 Scientific Advisory Group for Emergencies. Factors influencing covid-19 vaccine uptake among minority ethnic groups. 2021. https://assets.publishing.service.gov.uk/government/uploads/system/uploads/attachment_data/file/952716/s0979-factors-influencing-vaccine-uptake-minorityethnic-groups.pdf.

14 World Health Organization. WHO Director-General's opening remarks at 148th session of the Executive Board. 2021. www.who.int/director-general/speeches/detail/who-director-general-sopening-remarks-at-148th-session-of-the-executive-board.

15 So AD, Woo J. Reserving coronavirus disease 2019 vaccines for global access: cross sectional analysis. BMJ 2020;371:m4750. doi: 10.1136/bmj.m4750 pmid: 33323376

16 World Wide Web Foundation. It's time to recognise internet access as a human right. 2020. https://webfoundation.org/2020/10/its-time-to-recognise-internet-access-as-a-human-right.

17 Hung C. Immunity passport: great idea or hard pass? Healthcare Leadership Blog. 2020. https://hcldr.wordpress.com/2020/11/29/immunity-passport-great-idea-or-hard-pass.

This article is made freely available for use in accordance with BMJ's website terms and conditions for the duration of the covid-19 pandemic or until otherwise determined by BMJ. You may use, download and print the article for any lawful, non-commercial purpose (including text and data mining) provided that all copyright notices and trade marks are retained. 